\title{
Comparative efficacy and safety of antibody induction therapy for the treatment of kidney: a network meta-analysis
}

\author{
Mingjie Shao ${ }^{1}$, Tingting Tian², Xinyan Zhu ${ }^{3}$, Yingzi Ming ${ }^{1}$, Yasuko Iwakiri ${ }^{4}$, Shaojun \\ $\mathbf{Y e}^{5}$ and Qifa $Y \mathbf{e}^{1,5}$ \\ ${ }^{1}$ Department of Transplant Center, Xiangya 3rd Hospital, Central South University, Changsha, China \\ ${ }^{2}$ Department of Radiology, Changsha 1st Hospital, Changsha, China \\ ${ }^{3}$ Digestive Disease, Dongfang Hospital, Tongji University, Shanghai, China \\ ${ }^{4}$ Department of Internal Medicine, Section of Digestive Diseases, Yale University School of Medicine, New Haven, Connecticut, \\ USA \\ ${ }^{5}$ Department of Transplant, Zhongnan Hospital, Wuhan University, Wuhan, China \\ Correspondence to: Qifa Ye, email: yqf_china@163.com \\ Shaojun Ye, email: $86987100 @ q q . c o m$
}

Keywords: alemtuzumab, ATG, IL-2RAs, kidney transplantation, network meta-analysis

Received: April 04, $2017 \quad$ Accepted: July 12, $2017 \quad$ Published: August 02, 2017

Copyright: Shao et al. This is an open-access article distributed under the terms of the Creative Commons Attribution License 3.0 (CC BY 3.0), which permits unrestricted use, distribution, and reproduction in any medium, provided the original author and source are credited.

\section{ABSTRACT}

To evaluate the efficacy and safety of antibody induction therapies in kidney transplantation. Systematic literature searches were undertaken using MEDLINE, Embase, and Cochrane Library database from 1980 to 2016. Randomized controlled trials (RCTs) comparing three antibody induction therapies (alemtuzumab, interleukin-2 receptor antibodies and antithymocyte globulin) between each other were identified. Bayesian network meta-analysis was used to combine both the direct and indirect evidence on treatment efficacy and its safety. Antibody induction therapy studies, comprising of 18 RCTs ( 3444 kidney transplant recipients), were included. Overall, alemtuzumab treatment was superior to the ATG group (OR: $0.49,95 \%$ CI: 0.32 to 0.71 ) and IL-2RAs group (OR: $0.36,95 \% \mathrm{CI}: 0.25$ to 0.52 ) for reducing the 1-year acute rejection in kidney transplant recipients. Although alemtuzumab treatment was nearly same with ATG group and IL-2RAs group in improving patient survival and renal function, it can reduce the adverse effects of cytomegalovirus infection more efficiently than ATG group (OR: $0.59,95 \% \mathrm{CI}: 0.32$ to 0.95 ) and IL-2RAs group (OR: $1.08,95 \%$ CI: 0.61 to 1.73 ). Alemtuzumab was not associated with increased other adverse effects. Alemtuzumab treatment is safe and effective for kidney transplant recipients. No serious adverse effects were observed in trials or in general populations.

\section{INTRODUCTION}

Organ transplantation has developed rapidly since the introduction of immunosuppression drugs since the 1950s. Acute rejection (AR) is the continuously serious event that impacts long-term allograft survivals [1]. During the past 15 years of renal transplantation, many new induction factors were developed to decrease the incidence and adverse effects of acute rejection. For instance, the first category agent includes the interleukin-2 receptor antibodies (IL-2RAs), such as basiliximab and daclizumab, and non-depleting monoclonal antibodies. They directly target the CD25 epitope on T cells to limit acute injection and control adverse effects, when combined with CNI-based maintenance [2-4]. The second category is the depleting polyclonal antibody antithymocyte globulin (ATG) (Thymoglobulin) containing antibodies that target a majority of peripheral blood monocytes epitopes, with preventive effect on perfusion and preservation injury and chronic rejection, and that can reverse acute rejection [5-7] The third kind, alemtuzumab (Campath-1H) named humanized anti-CD52 monoclonal antibody, 
depletes T cells and other lymphocytes more powerfully, thereby decreasing the dosage of immunosuppressants significantly [8-10].

It has been reported that nearly $70 \%$ of renal transplant recipients have received induction therapy with either IL-2RAs or ATG in the past decade [11]. More and more transplant centers have used alemtuzumab as an antibody induction during solid organ transplantation recently [12]. Studies of comparing the effectiveness and safety of these four agents using traditional metaanalysis have been reported [13-21]. In 2006, Morris [22] discussed alemtuzumab in solid organ transplantation in his systematic review. However, it was performed in only two randomized controlled trials (RCTs) containing few numbers of patients. W.-J. Hao [23] tested effectiveness and safety of Alemtuzumab and Daclizumab compared to ATG, and Xin Zhang [24] and Robert D. Morgan [25] did similar studies by comparing the Alemtuzumab with ATG and IL-2RAs. Although plenty of efforts have been made, the optimal agents for induction use are still unmet.

Nowadays, more and more people have used network meta-analysis to evaluate the relative effectiveness of different treatments and combine evidence across selected RCTs. As we know, direct (trials compared alemtuzumab with basiliximab or daclizumab directly) and indirect estimates (trials compared alemtuzumab with basiliximab or daclizumab via ATG group) exist in the meta-analysis. Two estimates can be combined by the network meta-analysis then a compounded effect size was calculated as the weighted mean to evaluate the direct and indirect evidence [26]. Network meta-analysis is better than traditional meta-analysis for it can predict information from some comparisons which have no clinical trial data available. In our case, the exactness of the direct estimate was improved and the width of the confidence intervals was shortened by the indirect evidence [27].

Considering the increased numbers of new trials, this manuscript intends to compare the treatment outcome of alemtuzumab with other induction drugs such as basiliximab, daclizumab and ATG in kidney transplant for all RCTs using the Bayesian network meta-analysis [28-31]. Our study will provide useful information for transplant physicians.

\section{RESULTS}

\section{Characteristics of studies}

Figure 1 showed how we select the required trials in this study. In the original database and website searching we found 4380 records and then we discarded the duplicates, 2630 records were left. After 2443 records were eliminated, then 187 full-text were used for quantification. At last, 23 studies were included in this research [13-17, 19, 20, 32-47]. The details of the selected studies were listed in Table 1. Nine studies were identified as low quality (score $\leq 3$ ), and the rest were medium quality (scored 4 or 5) which can be seen in the methodological quality assessment (Table 2). 3444 kidney transplant recipients from eighteen studies were available for network meta-analysis [14-17, 20, 32-44]. Figure 2 was the comparisons structure of the primary outcomes.

\section{1- year AR}

Both network meta-analysis and traditional metaanalysis were summarized in Table 3. 1-year AR in alemtuzumab group is significantly lower compared to the ATG group (Ia, OR: $0.49,95 \%$ CI: 0.32 to 0.71 ) and IL-2RAs group (IIIa, OR: $0.36,95 \%$ CI: 0.25 to 0.52 ), and IL-2RAs showed a significantly higher 1-year AR compared to the ATG group (IIa, OR: 1.35, 95\% CI: 1.01 to 1.78). Direct and indirect estimates were consistent in this network meta-analysis. The posterior mean residual deviance is 20.8 (20 data points) indicating adequate fit. We can see the distribution of probability of each drug in Figure 3A. Alemtuzumab is possibly to be the most preferred treatment $(0 \%)$ when compared with ATG $(51 \%)$ and the IL-2RAs group (99\%), and the result of traditional meta-analysis was similar (Table 3 ). Heterogeneity among the three groups $\left(P, I^{2}\right)$ showed no significant different and publication bias also did not exist among these studies.

\section{1- year patient death}

Table 3 also indicated the results of network metaanalysis for the 1-year patient death. It showed that there was no significant difference among three groups-the alemtuzumab group and ATG group (Ib, OR: 2.29, 95\% CI: 0.51 to 6.20 ), the IL-2RAs group and ATG group (IIIb, OR: $1.71,95 \%$ CI: 0.70 to 3.94 ), the alemtuzumab group and IL-2RAs group (IIb, OR: 1.47, 95\% CI: 0.29 to 3.65). Evidence of inconsistency between the direct and indirect evidence cannot be seen. The posterior mean residual deviance is 18.7 ( 15 data points) which means good fit to data. Distribution of probability of each induction position can be seen in Figure 3B. From the result of the rank, we found that ATG is most likely $(14.5 \%)$ to be the preferred treatment for 1-year patient death, followed by IL-2RAs (60\%) and the alemtuzumab group (75.5\%). The traditional meta-analysis corroborated with similar results except for the comparison between the alemtuzumab group and ATG group. However, no significant heterogeneity among the three direct comparisons was found. Based on the $P$ values of Beggs' test, publication bias was not significant.

\section{1- year renal function}

We can see the results of network meta-analysis for 1-year renal function from the Table 3. No significant difference can be inferred between the alemtuzumab 
group and ATG group (Ib, SMD: $-0.18,95 \% \mathrm{CI}:-0.57$ to 0.15 ), between the IL-2RAs group and ATG group (IIId, SMD: -0.15 , 95\% CI: -0.37 to 0.09 ) and between the alemtuzumab group and IL-2RAs group (IId, SMD: -0.03 , $95 \% \mathrm{CI}:-0.42$ to 0.29 ). No evidence of inconsistency between the direct and indirect evidence was displayed. A posterior mean residual deviance of 13.8 (10 data points) shows an adequate fit. Distribution of probability of each drug was illustrated in Figure 3C. We can conclude that ATG is mostly the best treatment $(11 \%)$ for 1 -year renal function, then alemtuzumab (69\%) and the IL-2RAs group $(70 \%)$ based the result of the posterior probability values. The traditional meta-analysis also showed same results. Moreover, no significant heterogeneity was found among the three direct comparisons. There was no publication bias according to the $P$ values of Beggs' test.

\section{Adverse effects}

The harmful effects of induction mainly include delayed graft function (DGF), graft loss, cytomegalovirus (CMV) infection, malignancy, and new-onset diabetes mellitus after transplantation (NODAT). Among these five kinds of adverse effects, we only found significant difference appeared in CMV infection from Table 3 while others had no significant difference. A significantly lower CMV infection in alemtuzumab group when compared with the ATG group (Ig, OR: 0.59, 95\% CI: 0.32 to 0.95 ), also IL-2RAs achieved a significantly lower CMV infection compared with the ATG group (IIIg, OR: $0.55,95 \%$ CI: 0.38 to 0.78 ). No significant difference in alemtuzumab group was seen when compared with the IL-2RAs group (IIg, OR: 1.08 , 95\% CI: 0.61 to

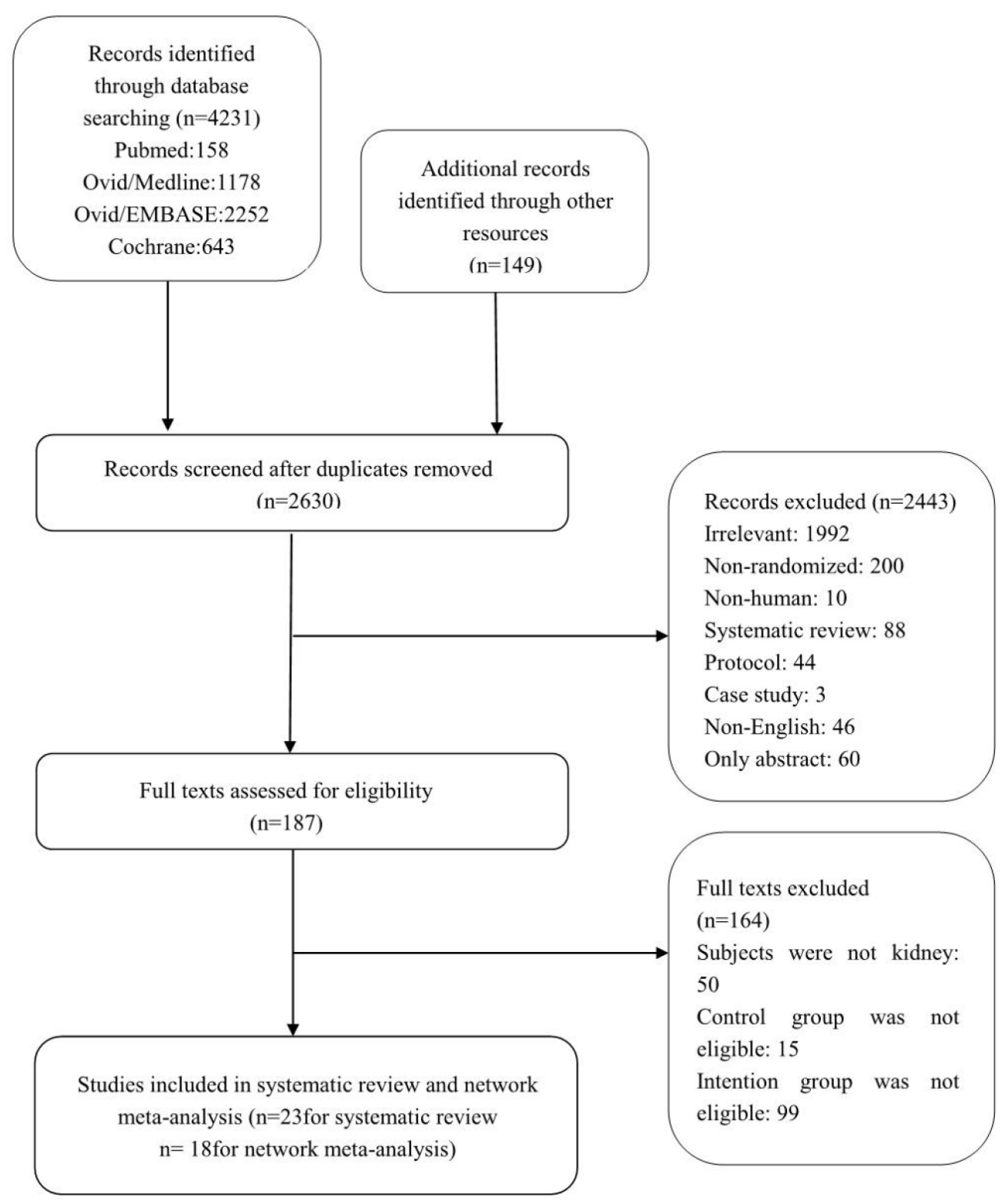

Figure 1: Flowchart for the selection of included trials. 
Table 1: Characteristics of the included studies

\begin{tabular}{|c|c|c|c|c|c|c|c|c|c|}
\hline Study & Group & No. & Age & Gender & $\begin{array}{l}\text { Dosage, duration of } \\
\text { induction }\end{array}$ & Follow-up & $\begin{array}{l}\text { Immunology } \\
\text { risk }\end{array}$ & $\begin{array}{l}\text { Donor } \\
\text { type }\end{array}$ & $\begin{array}{l}\text { Maintance } \\
\text { immunosupressive }\end{array}$ \\
\hline \multirow{2}{*}{$\begin{array}{l}\text { Matthew P WS } \\
\text { [38] } \\
2013\end{array}$} & G1:Ale & 58 & $46 \pm 14$ & $16 / 42$ & $30 \mathrm{mg}$ intraoperative & \multirow[t]{2}{*}{1 year } & \multirow[t]{2}{*}{ Low } & \multirow[t]{2}{*}{$\begin{array}{l}\mathrm{DD} \\
\mathrm{LD}\end{array}$} & Tac \\
\hline & G2:Bas & 58 & $49 \pm 14$ & $12 / 46$ & $20 \mathrm{mg}$ days 0,4 & & & & $\mathrm{Tac}+\mathrm{MMF}$ \\
\hline \multirow[t]{2}{*}{$\begin{array}{l}\text { The } 3 \mathrm{C} \text { group } \\
\text { [39] } \\
2014\end{array}$} & G1:Ale & 426 & $52.1 \pm 13.3$ & $277 / 149$ & $\begin{array}{l}30 \mathrm{mg} \text { after reperfusion } \\
\text { and } 24 \mathrm{~h} \text {, } \\
\text { age }>60 \text { y only one dose }\end{array}$ & \multirow[t]{2}{*}{1 year } & \multirow[t]{2}{*}{$\begin{array}{l}\text { Low } 96 \% \\
\text { High } 4 \%\end{array}$} & \multirow[t]{2}{*}{$\begin{array}{l}\text { DD } \\
\text { LD }\end{array}$} & low Tac + MMF \\
\hline & G2:Bas & 426 & $51.8 \pm 13.3$ & $275 / 151$ & $20 \mathrm{mg}$ days 0,4 & & & & $\begin{array}{l}\text { Standard } \\
\text { Tac+MMF+Pred }\end{array}$ \\
\hline \multirow{2}{*}{$\begin{array}{l}\text { Kakit Chan [40] } \\
2011\end{array}$} & G1:Ale & 82 & $47.3 \pm 13.4$ & $54 / 28$ & $30 \mathrm{mg}$ on return & \multirow[t]{2}{*}{2 year } & \multirow{2}{*}{$\begin{array}{l}\text { High and } \\
\text { Low }\end{array}$} & \multirow{2}{*}{$\begin{array}{l}\text { DD, } \\
\text { LD }\end{array}$} & Tac \\
\hline & $\mathrm{G} 2: \mathrm{Dac}$ & 41 & $47.0 \pm 10.6$ & $27 / 14$ & $2 \mathrm{mg} / \mathrm{kg}$ on return, $14 \mathrm{~d}$ & & & & Tac+MMF \\
\hline \multirow{2}{*}{$\begin{array}{l}\text { Alan C.Farney } \\
{[14]} \\
2009\end{array}$} & G1:Ale & 85 & $51 \pm 12$ & 1 & $30 \mathrm{mg}$ intraoperative & \multirow[t]{2}{*}{2 year } & \multirow[t]{2}{*}{$\begin{array}{l}\text { High and } \\
\text { Low }\end{array}$} & \multirow[t]{2}{*}{$\begin{array}{l}\text { DD } \\
\text { LD }\end{array}$} & \multirow[t]{2}{*}{$\begin{array}{l}\text { Tac/ } \\
\text { CsA+MMF+Pred }\end{array}$} \\
\hline & G2:rATG & 95 & $49 \pm 13$ & 1 & $1.5 \mathrm{mg} / \mathrm{kg}$ day $2,4,6$ & & & & \\
\hline \multirow{2}{*}{$\begin{array}{l}\text { Philip G.Thomas } \\
{[15]} \\
2007\end{array}$} & G1:Ale & 11 & $43.5 \pm 4.1$ & $6 / 5$ & $30 \mathrm{mg}$ pre-reperfusion & \multirow[t]{2}{*}{$\begin{array}{l}\text { 337day } \\
\text { 127 611day }\end{array}$} & \multirow[t]{2}{*}{ High } & \multirow[t]{2}{*}{ DD } & \multirow[t]{2}{*}{ Tac + MMF + Pred } \\
\hline & G2:ATG & 8 & $47.1 \pm 4.2$ & $2 / 6$ & $1.5 \mathrm{mg} / \mathrm{kg}$ for 4 days & & & & \\
\hline \multirow{2}{*}{$\begin{array}{l}\text { Lu Tie-ming } \\
{[17]} \\
2011\end{array}$} & G1:Ale & 11 & $38.9 \pm 4.2$ & $5 / 6$ & $\begin{array}{l}15 \mathrm{mg} \text { before reperfusion } \\
\text { and } 24 \mathrm{~h} \text { after Tx }\end{array}$ & \multirow[t]{2}{*}{$\begin{array}{l}\text { 338day } \\
\text { 30 730day }\end{array}$} & \multirow[t]{2}{*}{ High } & \multirow[t]{2}{*}{1} & \multirow[t]{2}{*}{ Tac + MMF + Pred } \\
\hline & G2:ATG & 11 & $40.8 \pm 4.4$ & $4 / 7$ & $9 \mathrm{mg} / \mathrm{kg}$ before $\mathrm{Tx}$ & & & & \\
\hline \multirow{2}{*}{$\begin{array}{l}\text { Ramzi Abou- } \\
\text { Ayache [20] } \\
2008\end{array}$} & G1:ATG & 51 & $45 \pm 12$ & $35 / 16$ & $\begin{array}{l}2 \mathrm{mg} / \mathrm{kg} \text { before } \mathrm{Tx} ; 1 \mathrm{mg} / \\
\mathrm{kg} \text { day } 14\end{array}$ & \multirow[t]{2}{*}{1 year } & Low & DD & $\mathrm{CsA}+\mathrm{MMF}+$ Pred \\
\hline & G2:Dac & 50 & $44 \pm 12$ & $36 / 14$ & $\begin{array}{l}\text { 1-1.5 mg/kg after Tx,day } \\
4 \sim \text { day } 9\end{array}$ & & & & \\
\hline Nicole A.Pilch & G1:ATG & 102 & $52 \pm 13$ & $59 / 43$ & $1.5 \mathrm{mg} / \mathrm{kg}$ day $0 \sim 4$ & 1 year & High $40 \%$ & DD & Tac + MMF+Pred \\
\hline $\begin{array}{l}{[42]} \\
2014\end{array}$ & G2:IL2RA & 98 & $49 \pm 14$ & $62 / 36$ & $\begin{array}{l}\mathrm{Dac} 1 \mathrm{mg} / \mathrm{kg} \text { day } 0,7 ; \text { Bas } \\
20 \mathrm{mg} \text { day0, } 4\end{array}$ & & Low $60 \%$ & & \\
\hline Daniel C.Brenna & G1:ATG & 141 & $51.3 \pm 13.1$ & $79 / 62$ & $1.5 \mathrm{mg} / \mathrm{kg}$ day $0 \sim 4$ & 1 year & 1 & 1 & CsA+MMF+Pred \\
\hline $\begin{array}{l}{[43]} \\
2006\end{array}$ & $\mathrm{G} 2: \mathrm{Bas}$ & 137 & $49.7 \pm 13.0$ & $82 / 55$ & $20 \mathrm{mg}$ day 0,4 & & & & \\
\hline Michael & G1:ATG & 69 & $48.5 \pm 11.0$ & $39 / 30$ & $1.5 \mathrm{mg} / \mathrm{kg}$ day $0 \sim 3$ & & High & & Tac + MMF+Pred \\
\hline $\begin{array}{l}\text { J.Hanaway [16] } \\
2011\end{array}$ & G2:Ale & 234 & $48.0 \pm 13.0$ & $152 / 82$ & $30 \mathrm{mg}$ day 0 & 3 year & $\begin{array}{l}\text { Low and } \\
\text { High }\end{array}$ & $\begin{array}{l}\mathrm{DD} \\
\mathrm{LD}\end{array}$ & $\begin{array}{l}5 \text { days later } \\
\text { Tac+MMF }\end{array}$ \\
\hline & G3:Bas & 171 & $48.9 \pm 13.6$ & $113 / 58$ & $20 \mathrm{mg}$ day 0,4 & & Low & & \\
\hline Gaetano Ciancio & G1:ATG & 43 & $47.8 \pm 2.0$ & $29 / 14$ & $1 \mathrm{mg} / \mathrm{kg} / \mathrm{d}$ for 7 days & & & & Tac+MMF+Pred \\
\hline $\begin{array}{l}{[41]} \\
2014\end{array}$ & G2:Ale & 43 & $47.1 \pm 2.0$ & $28 / 15$ & $0.3 \mathrm{mg} / \mathrm{kg}$ day 0,4 & 95month & Most Low & $\begin{array}{l}\text { DD } \\
90\end{array}$ & Low-Tac+MMF \\
\hline & G3:Dac & 42 & $49.2 \pm 1.9$ & $25 / 17$ & $\begin{array}{l}1 \mathrm{mg} / \mathrm{kg} \text { day } 0,14,28, \\
42,56\end{array}$ & & & $\begin{array}{l}\text { LD } \\
38\end{array}$ & Tac + MMF + Pred \\
\hline $\begin{array}{l}\text { LauriE Kyllonen } \\
\text { [44] }\end{array}$ & G1:ATG & 50 & 47.8 & $14 / 39$ & $\begin{array}{l}9 \mathrm{mg} / \mathrm{kg} \text { before } \\
\text { reperfusion }\end{array}$ & 5 year & Low & $\mathrm{DD}$ & $\mathrm{CsA}+\mathrm{Aza}+$ Pred \\
\hline 2007 & G2:Bas & 50 & 45.5 & $27 / 31$ & $20 \mathrm{mg}$ day 0,4 & & & & \\
\hline $\begin{array}{l}\text { Yvon Lebranchu } \\
\text { [45] }\end{array}$ & G1:ATG & 50 & $45.8 \pm 10.8$ & $32 / 18$ & $1-1.5 \mathrm{mg} / \mathrm{kg}$ for $6-10$ days & 1 year & Low & $\mathrm{DD}$ & CsA+MMF+Pred \\
\hline 2002 & G2:Bas & 50 & $44.1 \pm 11.5$ & $36 / 14$ & $20 \mathrm{mg}$ day 0,4 & & & & \\
\hline Christian Noel & G1:ATG & 113 & $45.4 \pm 10.3$ & $52 / 61$ & $1.25 \mathrm{mg} / \mathrm{kg}$ day $0-7$ & 1 year & High & 1 & Tac + MMF + Pred \\
\hline & G2:Dac & 114 & $46.9 \pm 9.0$ & $59 / 55$ & $\begin{array}{l}1 \mathrm{mg} / \mathrm{kg} \text { day } 0,14,28, \\
42,56\end{array}$ & & & 1 & \\
\hline
\end{tabular}




\begin{tabular}{|c|c|c|c|c|c|c|c|c|c|}
\hline \multirow{2}{*}{$\begin{array}{l}\text { S.G. Tullius [47] } \\
2003\end{array}$} & G1:ATG & 62 & 48 & $35 / 27$ & $9 \mathrm{mg} / \mathrm{kg}$ day 0 & \multirow[t]{2}{*}{1 year } & \multirow{2}{*}{$\begin{array}{l}\text { Low and } \\
\text { high }\end{array}$} & $\backslash$ & \multirow[t]{2}{*}{ Tac + MMF + Pred } \\
\hline & G2:Bas & 62 & 48 & $33 / 29$ & $20 \mathrm{mg}$ day 0,4 & & & 1 & \\
\hline \multirow{2}{*}{$\begin{array}{l}\text { Hans Sollinger } \\
{[48]} \\
2001\end{array}$} & G1:ATG & 65 & $49.8 \pm 11.9$ & $42 / 23$ & $1.5 \mathrm{mg} / \mathrm{kg}$ day $0-14$ & \multirow[t]{2}{*}{1 year } & 1 & DD & \multirow[t]{2}{*}{$\mathrm{CsA}+\mathrm{MMF}+$ Pred } \\
\hline & G2:Bas & 70 & $44.5 \pm 13.7$ & $37 / 33$ & $20 \mathrm{mg}$ day 0,4 & & 1 & LD & \\
\hline \multirow{2}{*}{$\begin{array}{l}\text { Domingo } \\
\text { Herna'ndez [49] } \\
2007\end{array}$} & G1:ATG & 80 & $47 \pm 12$ & $59 / 21$ & $1-1.5 \mathrm{mg} / \mathrm{kg}$ day $0-6$ & \multirow[t]{2}{*}{2 year } & 1 & $\backslash$ & $\mathrm{CsA}+\mathrm{Aza}+$ Pred \\
\hline & G2:Bas & 80 & $48 \pm 14$ & $50 / 30$ & $20 \mathrm{mg}$ day 0,4 & & 1 & $\backslash$ & $\begin{array}{l}\text { Low } \\
\text { CsA+MMF+Pred }\end{array}$ \\
\hline \multirow{2}{*}{$\begin{array}{l}\text { Georges Mourad } \\
{[50]} \\
2004\end{array}$} & G1:ATG & 53 & $45.4 \pm 12.7$ & $32 / 21$ & $1 \mathrm{mg} / \mathrm{kg}$ day 0,1 & \multirow[t]{2}{*}{1 year } & \multirow[t]{2}{*}{ Low } & \multirow[t]{2}{*}{$\mathrm{DD}$} & \multirow[t]{2}{*}{$\mathrm{CsA}+\mathrm{MMF}+\mathrm{Pred}$} \\
\hline & G2:Bas & 52 & $45.3 \pm 12.4$ & $30 / 22$ & $20 \mathrm{mg}$ day 0,4 & & & & \\
\hline \multirow{2}{*}{$\begin{array}{l}\text { Min Jeong } \mathrm{Kim}^{\dagger} \\
{[51]} \\
2008\end{array}$} & G1:ATG & 11 & $52(39-68)$ & $2 / 9$ & $\begin{array}{l}9 \mathrm{mg} / \mathrm{kg} \text { day } 0 ; 3 \mathrm{mg} / \mathrm{kg} / \mathrm{d} \\
\text { for } 4 \text { day }\end{array}$ & \multirow[t]{2}{*}{2 year } & \multirow[t]{2}{*}{ High } & \multirow[t]{2}{*}{ DD } & \multirow[t]{2}{*}{$\mathrm{CsA}+\mathrm{MMF}+$ Pred } \\
\hline & G2:Dac & 11 & $51(34-60)$ & $4 / 7$ & $\begin{array}{l}1 \mathrm{mg} / \mathrm{kg} \text { day } 0,14,28, \\
42,56\end{array}$ & & & & \\
\hline \multirow{2}{*}{$\begin{array}{l}\text { Alan Farney } \dagger \\
{[13]} \\
2008\end{array}$} & G1:Ale & 32 & $\backslash$ & $\backslash$ & $30 \mathrm{mg}$ intraoperative & \multirow[t]{2}{*}{2 year } & \multirow{2}{*}{$\begin{array}{l}\text { Low and } \\
\text { High }\end{array}$} & \multirow{2}{*}{$\begin{array}{l}\mathrm{DD} \\
\mathrm{LD}\end{array}$} & \multirow{2}{*}{$\begin{array}{l}\mathrm{Tac} / \\
\mathrm{CsA}+\mathrm{MMF}+\text { Pred }\end{array}$} \\
\hline & G2:ATG & 45 & $\backslash$ & $\backslash$ & $1.5 \mathrm{mg} / \mathrm{kg}$ day $2,4,6$ & & & & \\
\hline \multirow{3}{*}{$\begin{array}{l}\text { Gaetano } \\
\text { Ciancio† [52] } \\
2005\end{array}$} & G1:ATG & 30 & $49.3 \pm 2.5$ & $19 / 11$ & $1 \mathrm{mg} / \mathrm{kg} / \mathrm{d}$ for $7 \mathrm{~d}$ ays & \multirow{3}{*}{$27 \mathrm{mo}$} & \multirow{3}{*}{ Most Low } & \multirow{3}{*}{$\mathrm{DD}$} & Tac+MMF+Pred \\
\hline & G2:Ale & 30 & $50.2 \pm 2.1$ & $19 / 11$ & $0.3 \mathrm{mg} / \mathrm{kg}$ day 0,4 & & & & Low-Tac+MMF \\
\hline & G3:Dac & 30 & $49.9 \pm 2.4$ & $18 / 12$ & $\begin{array}{l}1 \mathrm{mg} / \mathrm{kg} \text { day } 0,14,28, \\
42,56\end{array}$ & & & & Tac+MMF+Pred \\
\hline \multirow{3}{*}{$\begin{array}{l}\text { Gaetano } \\
\text { Ciancio† [19] } \\
2010\end{array}$} & G1:ATG & 13 & $44.5 \pm 13.1$ & $10 / 3$ & $1 \mathrm{mg} / \mathrm{kg} / \mathrm{d}$ for 7 days & \multirow{3}{*}{3 year } & \multirow{3}{*}{ Low } & \multirow{3}{*}{ LD } & Tac+MMF+Pred \\
\hline & G2:Ale & 13 & $40.0 \pm 3.7$ & $9 / 4$ & $0.3 \mathrm{mg} / \mathrm{kg}$ day 0,4 & & & & Low-Tac+MMF \\
\hline & G3:Dac & 12 & $47.2 \pm 2.8$ & $7 / 5$ & $\begin{array}{l}1 \mathrm{mg} / \mathrm{kg} \text { day } 0,14,28, \\
42,56\end{array}$ & & & & Tac+MMF+Pred \\
\hline \multirow{2}{*}{$\begin{array}{l}\text { Karen L. } \\
\text { Hardinger† [53] } \\
2009\end{array}$} & G1:ATG & 141 & $\backslash$ & $\backslash$ & $1.5 \mathrm{mg} / \mathrm{kg}$ for 5 days & \multirow[t]{2}{*}{1 year } & High and & DD & $\mathrm{CsA}+\mathrm{MMF}+$ Pred \\
\hline & G2:Bas & 137 & 1 & 1 & $20 \mathrm{mg}$ day 0,4 & & Low & & \\
\hline
\end{tabular}

Ale: alemtuzumab; Bas: basiliximab; Dac: daclizumab; Tx: transplantation; DD: deceased donors; LD: living donors; Tac: tacrolimus; CsA: cyclosporine; MMF: Mycophenolate Mofetil; Pred: prednisone; Aza: azathioprine.

†Only data for the whole trial is available.

\section{Table 2: Methodological evaluation of included studies}

\begin{tabular}{lccccc}
\hline Study & Randomized method & Concealment allocation & Blinding method & Follow-up & Score \\
\hline Matthew P WS [38] & 2 & 2 & 0 & 1 & 5 \\
The 3C group [39] & 2 & 1 & 0 & 1 & 4 \\
Kakit Chan [40] & 2 & 2 & 0 & 1 & 5 \\
Alan C.Farney [14] & 1 & 1 & 1 & 1 & 4 \\
Philip G.Thomas [15] & 1 & 1 & 0 & 1 & 3 \\
Lu Tie-ming [17] & 1 & 0 & 0 & 1 & 2 \\
Ramzi Abou-Ayache [20] & 1 & 0 & 0 & 1 & 2 \\
Nicole A.Pilch [42] & 1 & 0 & 0 & 1 & 2 \\
Daniel C.Brenna [43] & 2 & 2 & 0 & 1 & 5 \\
Michael J.Hanaway [16] & 2 & 2 & 0 & 1 & 5 \\
Gaetano Ciancio [41] & 2 & 1 & 0 & 1 & 4 \\
LauriE Kyllonen [44] & 2 & 2 & 0 & 1 & 5 \\
Yvon Lebranchu [45] & 1 & 1 & 0 & 1 & 3 \\
Christian Noel [46] & 1 & 1 & 0 & 1 & 3 \\
S.G. Tullius [47] & 1 & 1 & 0 & 1 & 3 \\
Hans Sollinger [48] & 1 & 1 & 0 & 1 & 5 \\
Domingo Herna'ndez [49] & 2 & 2 & 0 & 1 & 3 \\
Georges Mourad [50] & 1 & 1 & 0 & 1 \\
\hline
\end{tabular}


1.73). It showed no evidence of inconsistency between the direct and indirect evidence. The posterior mean residual deviance of 23.6 (20 data points) in the model demonstrated an adequate fit. Figure 3D displayed the arrangement of a probability of each drug ranking. From the ranking, it can be inferred that IL-2RAs is most promising $(21.5 \%)$ to be the best drug for CMV infection, followed by alemtuzumab (29\%) and the ATG group $(99.5 \%)$. The traditional meta-analysis supported this result too. However, no significant heterogeneity was found in the three direct comparisons. Based on the $P$ values of Beggs' test, publication bias was nonsignificant among different kinds of studies. What's more, there was no significant difference among the three groups regarding the incidence of other adverse effects.

\section{DISCUSSION}

Antibody induction therapy is accepted as an important part of acquiring the best short and long-term results after the organ transplant [48]. Since the best induction agent has not yet to be established in clinical work, most of the randomized cohort studies focusing on comparison of alemtuzumab and other single induction agents (daclizumab, basiliximab, or ATG). Most of these studies have reported similar or even better outcome from first biopsy-proven acute rejection (BPAR) incidence in the alemtuzumab group with no unfavorable effects on renal function or graft survival [14-16, 25, 34, 49-51].

Three review papers published in 2012 involving six, ten and nine randomized controlled studies respectively came to a similar conclusion that alemtuzumab induction could be better induction agent in kidney transplantation due to it reduces the risk of AR but shares the similar incidence of other efficacy outcomes (graft loss, DGF, and graft/patient death). With gaining of evidence, this network meta-analysis certified that alemtuzumab induction is the most favored induction regarding therapeutic effects without significant adverse effects.

Our study, for the first time, reviewed three kinds of induction therapy (alemtuzumab, IL-2RAs and ATG) after kidney transplantation using network meta-analysis to compile both direct and indirect evidence of therapeutic and adverse effects. In the present network meta-analysis, alemtuzumab is thought to be better than traditional induction antibodies in preventing 1year AR. Changes in rejection rates among the groups, we found, did not result in significant differences in the survival of patients or allografts. DGF and infection are still the two major posttransplant issues that have an ill effect on both qualities of

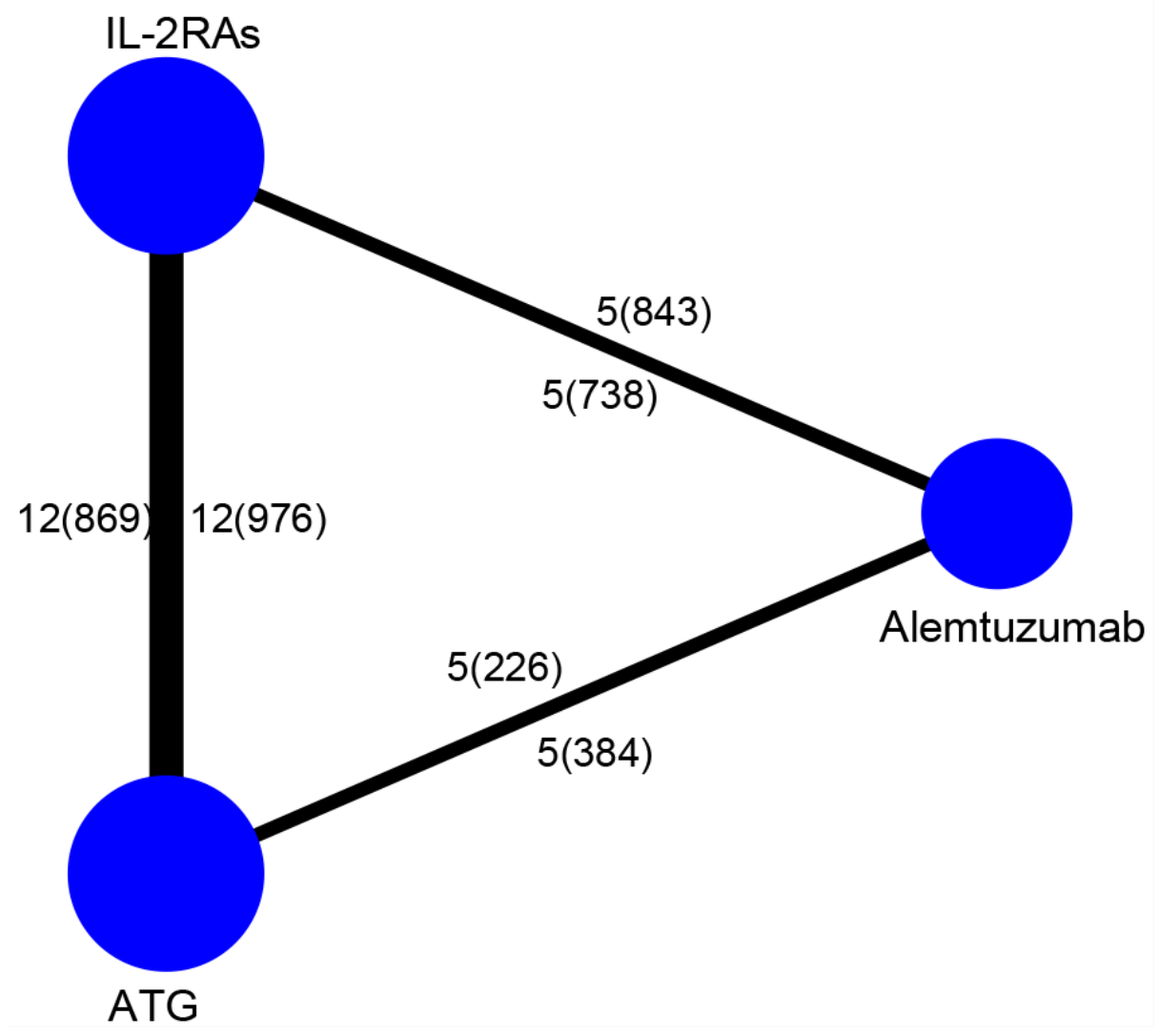

Figure 2: Structure of treatments and direct comparisons of network. The lines between two nodes indicate that direct compared randomized trials. The weight of the lines is quantified to the number of trials used for comparison. Trials amount was presented with numbers outside while patients amount was inside the braces. 
Table 3: Results of network meta-analysis and traditional meta-analysis

\begin{tabular}{|c|c|c|c|c|c|}
\hline & Comparison & $\begin{array}{l}\text { Network meta-analysis, } \\
\text { OR/SMD* (95\% CI) }\end{array}$ & $\begin{array}{l}\text { Traditional meta-analysis, } \\
\text { OR/SMD* (95\% CI) }\end{array}$ & $\begin{array}{l}\text { Heterogeneity } \\
\text { (P/I2) }\end{array}$ & $\begin{array}{c}\text { Publication bias } \\
\text { (P value of Beggs' test) }\end{array}$ \\
\hline I & Ale vs ATG & & & & \\
\hline $\mathrm{a}$ & $1 \mathrm{yr} A R$ & $0.49(0.32,0.71)$ & $0.46(0.27,0.78)$ & $0.64 / 0 \%$ & 0.734 \\
\hline b & 1yr Patient death & $2.29(0.51,6.20)$ & $0.90(0.36,2.26)$ & $0.80 / 0 \%$ & 1.000 \\
\hline $\mathrm{c}$ & 1yr Graft death & $1.73(0.50,4.02)$ & $0.90(0.36,2.26)$ & $0.80 / 0 \%$ & 1.000 \\
\hline d & Renal function & $-0.18(-0.57,0.15)^{*}$ & $-0.44(-1.12,0.25)^{*}$ & $0.14 / 54 \%$ & 0.734 \\
\hline $\mathrm{e}$ & DGF & $1.36(0.78,2.05)$ & $0.88(0.52,1.50)$ & $0.29 / 20 \%$ & 1.000 \\
\hline$f$ & Graft loss & $0.81(0.38,1.51)$ & $0.57(0.31,1.08)$ & $0.44 / 0 \%$ & 0.734 \\
\hline$g$ & CMV infection & $0.59(0.32,0.95)$ & $0.81(0.46,1.41)$ & $0.32 / 13 \%$ & 1.000 \\
\hline $\mathrm{h}$ & Malignance & $0.62(0.01,2.46)$ & $1.11(0.03,37.29)$ & $0.05 / 74 \%$ & 1.000 \\
\hline $\mathrm{i}$ & NODAT & $0.49(0.18,1.08)$ & $0.39(0.16,0.93)$ & $0.32 / 0 \%$ & 1.000 \\
\hline II & Anti-IL2r vs ATG & & & & \\
\hline $\mathrm{a}$ & $1 \mathrm{yr} A R$ & $1.35(1.01,1.78)$ & $1.38(1.07,1.78)$ & $0.69 / 0 \%$ & 0.533 \\
\hline $\mathrm{b}$ & 1yr Patient death & $1.71(0.70,3.94)$ & $1.11(0.72,1.71)$ & $0.64 / 0 \%$ & 0.108 \\
\hline $\mathrm{c}$ & 1yr Graft death & $1.18(0.59,2.29)$ & $1.11(0.72,1.71)$ & $0.64 / 0 \%$ & 0.174 \\
\hline $\mathrm{d}$ & Renal function & $-0.15(-0.37,0.09)^{*}$ & $-0.14(-0.26,0.01)^{*}$ & $0.09 / 47 \%$ & 0.118 \\
\hline $\mathrm{e}$ & DGF & $1.19(0.86,1.60)$ & $1.22(0.95,1.56)$ & $0.10 / 41 \%$ & 0.754 \\
\hline $\mathrm{f}$ & Graft loss & $0.93(0.58,1.44)$ & $0.95(0.67,1.35)$ & $0.46 / 0 \%$ & 1.000 \\
\hline $\mathrm{g}$ & CMV infection & $0.55(0.38,0.78)$ & $0.53(0.41,0.68)$ & $0.32 / 13 \%$ & 0.373 \\
\hline $\mathrm{h}$ & Malignance & $0.36(0.02,1.19)$ & $0.62(0.30,1.25)$ & $0.31 / 16 \%$ & 0.764 \\
\hline $\mathrm{i}$ & NODAT & $0.82(0.39,1.55)$ & $0.78(0.46,1.34)$ & $0.62 / 0 \%$ & 0.707 \\
\hline III & Ale vs anti-IL2r & & & & \\
\hline $\mathrm{a}$ & $1 \mathrm{yr} A R$ & $0.36(0.25,0.52)$ & $0.36(0.26,0.50)$ & $0.55 / 0 \%$ & 0.806 \\
\hline b & 1yr Patient death & $1.47(0.29,3.65)$ & $1.53(0.41,5.72)$ & $0.10 / 57 \%$ & 0.296 \\
\hline $\mathrm{c}$ & 1yr Graft death & $1.54(0.45,3.37)$ & $1.53(0.41,5.72)$ & $0.10 / 57 \%$ & 0.296 \\
\hline$d$ & Renal function & $-0.03(-0.42,0.29)^{*}$ & $0.15(-0.03,0.32)^{*}$ & $0.21 / 37 \%$ & 0.462 \\
\hline e & DGF & $1.15(0.71,1.65)$ & $1.31(1.01,1.70)$ & $0.98 / 0 \%$ & 0.734 \\
\hline $\mathrm{f}$ & Graft loss & $0.89(0.39,1.73)$ & $1.28(0.50,3.26)$ & $0.84 / 0 \%$ & 0.296 \\
\hline g & CMV infection & $1.08(0.61,1.73)$ & $0.94(0.43,2.03)$ & $0.07 / 54 \%$ & 0.806 \\
\hline $\mathrm{h}$ & Malignance & $2.66(0.07,13.56)$ & $1.02(0.53,1.96)$ & $0.56 / 0 \%$ & 1.000 \\
\hline $\mathrm{i}$ & NODAT & $0.61(0.27,1.21)$ & $0.61(0.33,1.12)$ & $0.81 / 0 \%$ & 0.089 \\
\hline
\end{tabular}

life and patient/graft survival [52, 53]. This meta-analysis certifies that alemtuzumab is similar when compared with traditional antibodies for preventing both DGF and post-transplant infection. Also, it shows no significant difference in the distribution of probability observed between the group treated with IL-2RA and alemtuzumab in CMV infection. Therefore, alemtuzumab treatment is safe and effective for kidney transplant recipients.

Nevertheless, the limitations of this study should be pointed out. Since it is impossible to blind induction treatment, it has to be an open-label trial. Because of the limited number of included trials, especially the comparative group of alemtuzumab vs ATG and IL2RAs vs ATG, there is no enough data for us to assess the effectiveness and safety of alemtuzumab in particular patient populations thoroughly, such as live versus deceased donors, or low- versus high-risk recipients. Also, our study has little power to detect 2 year or 3 year AR after transplant when the data is limited to outcomes that occur only during the first year post-transplant. Same situation also occurs in graft loss and patient survival.

\section{MATERIALS AND METHODS}

\section{Literature search}

We used many logic combinations of keywords and text words to search the Pubmed, Embase and Cochrane library as well as related interventions and randomized controlled trials till August 2016 (Supplementary Appendix 1). Also, we also searched the websites manually as follows: Current Controlled Trials, ClinicalTrials.gov 
and The World Health Organization International Clinical Trials Registry.

\section{Study selection}

The abstracts and full texts found were checked by two researchers independently. We resolved the disagreements by discussing and consulting to another researcher. Included criteria for the papers in the analysis were: (1) randomized controlled studies; (2) kidney transplant recipients with induction (3) studies referring to at least two of the following eligible inductions: alemtuzumab, IL-2RAs and ATG; (4) studies containing the main or adverse outcomes; (5) English literature.

\section{Quality assessment}

We used the modified Oxford score [54, 55] to evaluate the quality of methodology of included studies. Score 0 to 7 was given based on randomization, concealment allocation, blinding method and reporting of participant withdrawals.

\section{Outcome measures}

Our study was mainly to determine the effectiveness of patients and grafts outcomes and adverse outcomes by using the alemtuzumab, IL-2RAs and ATG induction respectively. The patients and grafts outcomes contain 1-year biopsy-proven acute rejection, 1-year patient death, 1-year renal function and DGF; the adverse outcomes include CMV infections, NODAT, malignancy and graft loss.

\section{Statistical analysis}

We compared the effectiveness of patients and grafts outcomes and adverse outcomes among three kinds of different induction therapy for kidney transplant recipients for the random effect of Bayesian network meta-analysis.
A
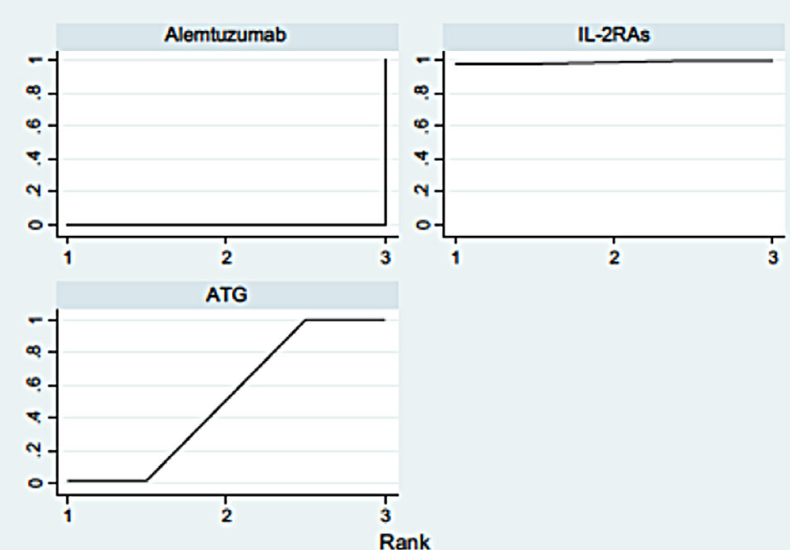

Graphs by Treatment

C
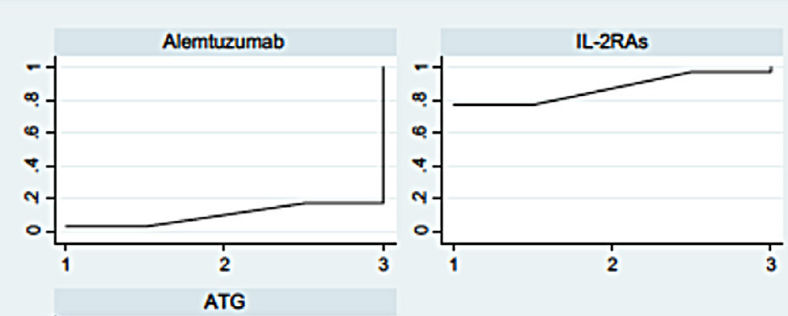

B
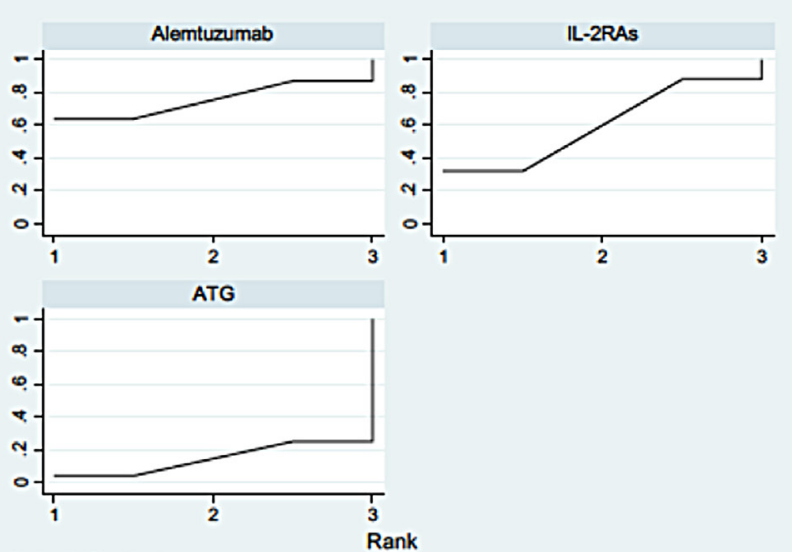

Graphs by Treatmen

D
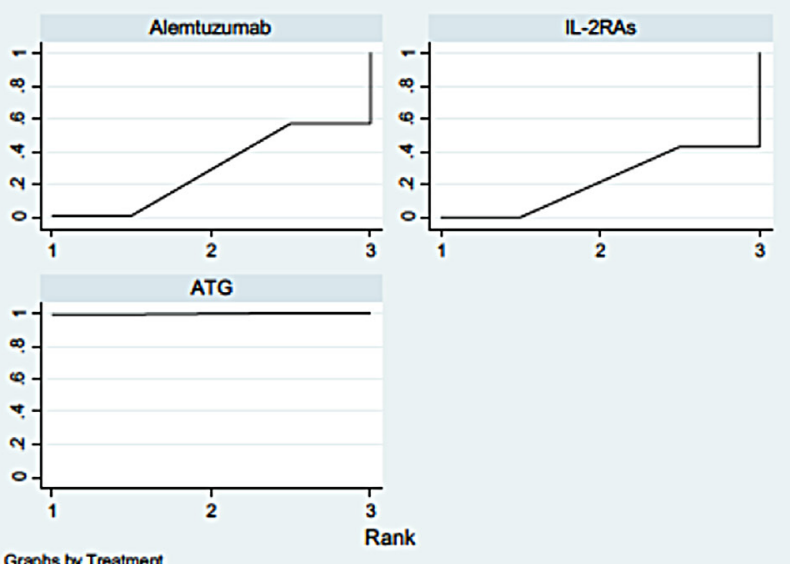

Graphs by Treatmert

Figure 3: Rankings for efficacy and safety. The graph displays effectiveness of each drug. X-axis shows the ranking of each drug (from the most effective to the least), Y-axis indicates the cumulative probability for each drug to be the most preferred drug. (A) 1 year acute rejection; (B) 1 year patient death; (C) 1 year renal function; (D) CMV infection. 
The network meta-analysis allows indirect comparisons of interventions among cohorts which the meta-analysis not. In our study, we used WinBUGS (version 1.4.2, MRC Biostatistics Unit, Cambridge, UK), R (version 3.1.2, The $\mathrm{R}$ Foundation for Statistical Computing) and STATA (version 12.0, StataCorp, College Station, TX) to perform the Bayesian network meta-analysis. Multi-arm trials often used codes of random effect models which can be seen in http://www.mtm.uoi.gr/ (Supplementary Appendix 2). Three Markov chains ran synchronously with different initial values which were chosen at random. Each of the three sets of initial values had 50,000 simulations generated, we discard the first 10,000 simulations for the burn-in period. The median of the posterior distribution was used to calculate the pooled effect sizes. We used the $2.5 \%$ and $97.5 \%$ of the posterior distribution named corresponding 95\% credible intervals same as the conventional $95 \%$ confidence intervals. For estimating the network inconsistency of every closed loop, absolute difference was calculated between the direct and indirect evidence. Loops showed statistically significant inconsistency if the lower CI extremity still far away the zero line [56]. The accuracy of the model was defined by calculating the posterior mean residual deviance. The number of data points of the model can be measured by the mean of the residual deviance, which means that the model fits the data adequately [57]. We ranked the treatments according to the degree of effectiveness based their posterior probabilities (first choice, second choice, third choice, etc.). The surface under the cumulative ranking (SUCRA) used for the measurement of probability values [58]. If the SUCRA is equal to 1 that means it is the most effective treatment. Otherwise, 0 means the most ineffective treatment.

The traditional meta-analysis was done with STATA (version 12.0, StataCorp, College Station, TX). Q statistics $(P<0.05$ was considered heterogeneous $)$ and $I^{2}$ statistics ( $I^{2} \geq 50 \%$ was considered heterogeneous) were used to test the heterogeneity. Begg's tests [59] were used to evaluate the publication bias, if the $P$ value was less than 0.5 then the publication bias was established.

\section{CONCLUSIONS}

This network meta-analysis indicated that alemtuzumab, interleukin-2 receptor antibodies and antithymocyte globulin, are all effective in improving survival and renal function of the patients. Overall, however, alemtuzumab showed a greater probability of being the preferred regimen in reducing the 1-year acute rejection and a significantly lower risk of cytomegalovirus infection when compared with interleukin-2 receptor antibodies and antithymocyte globulin. Therefore, our results suggest that alemtuzumab should be considered as the recommend antibody induction in the treatment of kidney transplantation. Further random control trials (RCTs) are needed to confirm this result.

\section{Abbreviations}

RCTs: randomized controlled trials; IL-2RAs: interleukin-2 receptor antibodies; ATG: antithymocyte globulin; AR: acute rejection; BPAR: biopsy-proven acute rejection; DGF: delayed graft function; NODAT: new-onset diabetes mellitus after transplantation; CMV: cytomegalovirus; SUCRA: surface under the cumulative ranking.

\section{Author contributions}

All authors have the right to use the data and are responsible for the truth and the accuracy of the data.

MJ Shao and QF Ye contributed to the study design and manuscript writing. TT Tian, YZ Ming and XY Zhu contributed to data collection. TT Tian and XY Zhu contributed to preparation and data analysis. Y.Iwakiri, SJ Ye and QF Ye contributed to the manuscript revision.

\section{ACKNOWLEDGMENTS}

Thanks for Chao Zeng and Jie Wei for guiding the methods and statistics.

\section{CONFLICTS OF INTEREST}

All the authors declared no competing interests.

\section{FUNDING}

This work was supported by the Major Program of National Natural Science Foundation of China (Nos. U1403223).

\section{REFERENCES}

1. Barr ML, Belghiti J, Villamil FG, Pomfret EA, Sutherland DS, Gruessner RW, Langnas AN, Delmonico FL. A report of the Vancouver Forum on the care of the live organ donor: lung, liver, pancreas, and intestine data and medical guidelines. Transplantation. 2006; 81:1373-85.

2. Vincenti F, Kirkman R, Light S, Bumgardner G, Pescovitz M, Halloran P, Neylan J, Wilkinson A, Ekberg H, Gaston R, Backman L, Burdick J. Interleukin-2-receptor blockade with daclizumab to prevent acute rejection in renal transplantation. Daclizumab Triple Therapy Study Group. N Engl J Med. 1998; 338:161-5.

3. Nashan B, Light S, Hardie IR, Lin A, Johnson JR. Reduction of acute renal allograft rejection by daclizumab. Daclizumab Double Therapy Study Group. Transplantation. 1999; 67:110-5.

4. Ciancio G, Burke GW, Suzart K, Roth D, Kupin W, Rosen A, Olson L, Esquenazi V, Miller J. Daclizumab induction, tacrolimus, mycophenolate mofetil and steroids as an 
immunosuppression regimen for primary kidney transplant recipients. Transplantation. 2002; 73: 1100.

5. Gaber AO, First MR, Tesi RJ, Gaston RS, Mendez R, Mulloy LL, Light JA, Gaber LW, Squiers E, Taylor RJ, Neylan JF, Steiner RW, Knechtle S, et al. Results of the double-blind, randomized, multicenter, phase III clinical trial of Thymoglobulin versus Atgam in the treatment of acute graft rejection episodes following renal transplant. Transplantation. 1998; 66:29-37.

6. Goggins WC, Pascual MA, Powelson JA, Magee C, Tolkoff-Rubin N, Farrell ML, Ko DS, Williams WW, Chandraker A, Delmonico FL, Auchincloss H, Cosimi AB. A prospective, randomized, clinical trial of intraoperative versus postoperative Thymoglobulin in adult cadaveric renal transplant recipients. Transplantation. 2003; 76:798-802.

7. Matas AJ, Kandaswamy R, Humar A, Payne WD, Dunn DL, Najarian JS, Gruessner RW, Gillingham KJ, McHugh LE, Sutherland DE. Long-term immunosuppression, without maintenance prednisone, after kidney transplantation. Ann Surg. 2004; 240:510-6.

8. Knechtle SJ, Pirsch JD, H Fechner J Jr, Becker BN, Friedl A, Colvin RB, Lebeck LK, Chin LT, Becker YT, Odorico JS, D'Alessandro AM, Kalayoglu M, Hamawy MM, et al. Campath-1H induction plus rapamycin monotherapy for renal transplantation: results of a pilot study. Am J Transplant. 2003; 3:722-30.

9. Ciancio G, Burke GW, Gaynor JJ, Mattiazzi A, Roohipour R, Carreno MR, Roth D, Ruiz P, Kupin W, Rosen A, Esquenazi V, Tzakis AG, Miller J. The use of Campath-1H as induction therapy in renal transplantation: preliminary results. Transplantation. 2004; 78:426-33.

10. Calne R, Friend P, Moffatt S, Bradley A, Hale G, Firth J, Bradley J, Smith K, Waldmann H. Prope tolerance, perioperative campath $1 \mathrm{H}$, and low-dose cyclosporin monotherapy in renal allograft recipients. Lancet. 1998; 351:1701-2.

11. Meier-Kriesche HU, Arndorfer JA, Kaplan B. Association of antibody induction with short- and long-term causespecific mortality in renal transplant recipients. J Am Soc Nephrol. 2002; 13:769-72.

12. Weaver TA, Kirk AD. Alemtuzumab. Transplantation. 2007; 84:1545-7.

13. Farney A, Sundberg A, Moore P, Hartmann E, Rogers J, Doares W, Jarrett A, Adams P, Stratta R. A randomized trial of alemtuzumab vs. anti-thymocyte globulin induction in renal and pancreas transplantation. Clin Transplant. 2008; 22:41-9.

14. Farney AC, Doares W, Rogers J, Singh R, Hartmann E, Hart L, Ashcraft E, Reeves-Daniels A, Gautreaux M, Iskandar SS, Moore P, Adams PL, Stratta RJ. A randomized trial of alemtuzumab versus antithymocyte globulin induction in renal and pancreas transplantation. Transplantation. 2009; 88:810-9.

15. Thomas PG, Woodside KJ, Lappin JA, Vaidya S, Rajaraman S, Gugliuzza KK. Alemtuzumab (Campath 1H) induction with tacrolimus monotherapy is safe for high immunological risk renal transplantation. Transplantation. 2007; 83:1509-12.
16. Hanaway MJ, Woodle ES, Mulgaonkar S, Peddi VR, Kaufman DB, First MR, Croy R, Holman J, INTAC Study Group. Alemtuzumab induction in renal transplantation. N Engl J Med. 2011; 364:1909-19.

17. Lü TM, Yang SL, Wu WZ, Tan JM. Alemtuzumab induction therapy in highly sensitized kidney transplant recipients. Chin Med J (Engl). 2011; 124:664-8.

18. Ciancio G, Burke GW, Gaynor JJ, Roth D, Kupin W, Rosen A, Cordovilla T, Tueros L, Herrada E, Miller J. A randomized trial of thymoglobulin vs. alemtuzumab (with lower dose maintenance immunosuppression) vs. daclizumab in renal transplantation at 24 months of followup. Clin Transplant. 2008; 22:200-10.

19. Ciancio G, Gaynor JJ, Roth D, Kupin W, Hanson L, Tueros L, Zarak A, Ruiz P, Burke GW 3rd. Randomized trial of thymoglobulin versus alemtuzumab (with lower dose maintenance immunosuppression) versus daclizumab in living donor renal transplantation. Transplant Proc. 2010; 42:3503-6.

20. Abou-Ayache R, Büchler M, Lepogamp P, Westeel PF, Le Meur Y, Etienne I, Lobbedez T, Toupance O, Caillard S, Goujon JM, Bergougnoux L, Touchard G. CMV infections after two doses of daclizumab versus thymoglobulin in renal transplant patients receiving mycophenolate mofetil, steroids and delayed cyclosporine A. Nephrol Dial Transplant. 2008; 23:2024-32.

21. Huurman VA, Kalpoe JS, van de Linde P, Vaessen N, Ringers J, Kroes AC, Roep BO, De Fijter JW. Choice of antibody immunotherapy influences cytomegalovirus viremia in simultaneous pancreas-kidney transplant recipients. Diabetes Care. 2006; 29:842-7.

22. Morris PJ, Russell NK. Alemtuzumab (Campath-1H): a systematic review in organ transplantation. Transplantation. 2006; 81:1361-7.

23. Hao WJ, Zong HT, Cui YS, Zhang Y. The Efficacy and Safety of Alemtuzumab and Daclizumab Versus Antithymocyte Globulin During Organ Transplantation A Meta-Analysis. Transplant Proc. 2012; 44:2955-60.

24. Zhang X, Huang H, Han S, Fu S, Wang L. Alemtuzumab induction in renal transplantation: a meta-analysis and systemic review. Transpl Immunol. 2012; 27: 63-8.

25. Morgan RD, O'Callaghan JM, Knight SR, Morris PJ. Alemtuzumab induction therapy in kidney transplantation: a systematic review and meta-analysis. Transplantation. 2012; 93:1179-88.

26. Cipriani A, Higgins JP, Geddes JR, Salanti G. Conceptual and technical challenges in network meta-analysis. Ann Intern Med. 2013; 159:130-7.

27. Bucher HC, Guyatt GH, Griffith LE, Walter SD. The results of direct and indirect treatment comparisons in metaanalysis of randomized controlled trials. J Clin Epidemiol. 1997; 50:683-91.

28. Caldwell DM, Ades AE, Higgins JP. Simultaneous comparison of multiple treatments: combining direct and indirect evidence. BMJ. 2005; 331:897-900. 
29. Caldwell DM, Welton NJ, Ades AE. Mixed treatment comparison analysis provides internally coherent treatment effect estimates based on overviews of reviews and can reveal inconsistency. J Clin Epidemiol. 2010; 63:875-82.

30. Lumley T. Network meta-analysis for indirect treatment comparisons. Stat Med. 2002; 21:2313-24.

31. Lu G, Ades AE. Combination of direct and indirect evidence in mixed treatment comparisons. Stat Med. 2004; 23:3105-24.

32. Welberry Smith MP, Cherukuri A, Newstead CG, Lewington AJ, Ahmad N, Menon K, Pollard SG, Prasad P, Tibble S, Giddings E, Baker RJ. Alemtuzumab induction in renal transplantation permits safe steroid avoidance with tacrolimus monotherapy a randomized controlled trial. Transplantation. 2013; 96:1082-8.

33. 3C Study Collaborative Group, Haynes R, Harden P, Judge P, Blackwell L, Emberson J, Landray MJ, Baigent C, Friend PJ. Alemtuzumab-based induction treatment versus basiliximabbased induction treatment in kidney transplantation (the 3C Study) a randomised trial. Lancet. 2014; 384:1684-90.

34. Chan K, Taube D, Roufosse C, Cook T, Brookes P, Goodall D, Galliford J, Cairns T, Dorling A, Duncan N, Hakim N, Palmer A, Papalois V, et al. Kidney transplantation with minimized maintenance alemtuzumab induction with tacrolimus monotherapy - an open label, randomized trial. Transplantation. 2011; 92:774-80.

35. Ciancio G, Gaynor JJ, Guerra G, Sageshima J, Chen L, Mattiazzi A, Roth D, Kupin W, Tueros L, Flores S, Hanson L, Vianna R, Burke GW 3rd. Randomized Trial of Three Induction Antibodies in Kidney Transplantation Long-Term Results. Transplantation. 2014; 97:1128-38.

36. Pilch NA, Taber DJ, Moussa O, Thomas B, Denmark S, Meadows HB, McGillicuddy JW, Srinivas TR, Baliga PK, Chavin KD. Prospective randomized controlled trial of rabbit antithymocyte globulin compared with IL-2 receptor antagonist induction therapy in kidney transplantation. Ann Surg. 2014; 259:888-93.

37. Brennan DC, Daller JA, Lake KD, Cibrik D, Del Castillo D, Thymoglobulin Induction Study Group. Rabbit antithymocyte globulin versus basiliximab in renal transplantation. N Engl J Med. 2006; 355:1967-77.

38. Kyllönen LE, Eklund BH, Pesonen EJ, Salmela KT. Single Bolus Antithymocyte Globulin Versus Basiliximab Induction in Kidney Transplantation With Cyclosporine Triple Immunosuppression Efficacy and Safety. Transplantation. 2007; 84:75-82.

39. Lebranchu Y, Bridoux F, Büchler M, Le Meur Y, Etienne I, Toupance O, Hurault de Ligny B, Touchard G, Moulin B, Le Pogamp P, Reigneau O, Guignard M, Rifle G. Immunoprophylaxis with Basiliximab Compared with Antithymocyte Globulin in Renal Transplant Patients Receiving MMF-containing Triple Therapy. Am J Transplant. 2002; 2:48-56.

40. Noël C, Abramowicz D, Durand D, Mourad G, Lang P, Kessler M, Charpentier B, Touchard G, Berthoux F, Merville P,
Ouali N, Squifflet JP, Bayle F, et al. Daclizumab versus Antithymocyte Globulin in High-Immunological-Risk Renal Transplant Recipients. J Am Soc Nephrol. 2009; 20:1385-92.

41. Tullius SG, Pratschke J, Strobelt V, Kahl A, Reinke P, May G, Frei U, Neuhaus P. ATG versus basiliximab induction therapy in renal allograft recipients receiving a dual immunosuppressive regimen: one-year results. Transplant Proc. 2003; 35:2100-1.

42. Sollinger H, Kaplan B, Pescovitz MD, Philosophe B, Roza A, Brayman K, Somberg K. Basiliximab versus antithymocyte globulin for prevention of acute renal allograft rejection. Transplantation. 2001; 72:1915-9.

43. Hernández D, Miquel R, Porrini E, González-Posada JM, Hortal L, Checa MD, Rodríguez A, García JJ, Rufino M, Torres A. Randomized Controlled Study Comparing Reduced Calcineurin Inhibitors Exposure Versus Standard Cyclosporine-Based Immunosuppression. Transplantation. 2007; 84:706-14.

44. Mourad G, Rostaing L, Legendre C, Garrigue V, Thervet E, Durand D. Sequential Protocols using Basiliximab versus Anti-Thymocyte Globulins in Renal-Transplant Patients Receiving Mycophenolate Mofetil and Steroids. Transplantation. 2004; 78:584-90.

45. Kim MJ, Tsinalis D, Franz S, Binet I, Gürke L, Mihatsch MJ, Steiger J, Thiel G, Dickenmann M. ATG-Fresenius or daclizumab induction therapy in immunologically high risk kidney recipients: a prospective randomized pilot trial. Ann Transplant. 2008; 13:21-7.

46. Ciancio G, Burke GW, Gaynor JJ, Carreno MR, Cirocco RE, Mathew JM, Mattiazzi A, Cordovilla T, Roth D, Kupin W, Rosen A, Esquenazi V, Tzakis AG, Miller J. A randomized trial of three renal transplant induction antibodies early comparison of tacrolimus, mycophenolate mofetil, and steroid dosing, and newer immune-monitoring. Transplantation. 2005; 80:457-65.

47. Hardinger KL, Brennan DC, Schnitzler MA. Rabbit antithymocyte globulin is more beneficial in standard kidney than in extended donor recipients. Transplantation. 2009; 87:1372-6.

48. Woodle ES, Alloway RR, Buell JF, Alexander JW, Munda R, Roy-Chaudhury P, First MR, Cardi M, Trofe J. Multivariate analysis of risk factors for acute rejection in early corticosteroid cessation regimens under modern immunosuppression. Am J Transplant. 2005; 5:2740-4.

49. Vathsala A, Ona ET, Tan SY, Suresh S, Lou HX, Casasola CB, Wong HC, Machin D, Chiang GS, Danguilan RA, Calne R. Randomized trial of alemtuzumab for prevention of graft rejection and preservation of renal function after kidney transplantation. Transplantation. 2005; 80:765-74.

50. Margreiter R, Klempnauer J, Neuhaus P, Suresh S, Lou HX, Casasola CB, Wong HC, Machin D, Chiang GS, Danguilan RA, Calne R. Alemtuzumab (Campath-1H) and tacrolimus monotherapy after renal transplantation: results of a prospective randomized trial. Am J Transplant. 2008; 8:1480-5. 
51. Cherukuri A, Salama AD, Carter C, Smalle N, McCurtin R, Hewitt EW, Hernandez-Fuentes M, Clark B, Baker RJ. An analysis of lymphocyte phenotype after steroid avoidance with either alemtuzumab or basiliximab induction in renal transplantation. Am J Transplant. 2012; 12:919-31.

52. Kim HC, Hwang EA, Kang MJ, Han SY, Park SB, Park KK. $\mathrm{BK}$ virus infection in kidney transplant recipients. Transplant Proc. 2004; 36:2113-5.

53. Quiroga I, McShane P, Koo DD, Gray D, Friend PJ, Fuggle S, Darby C. Major effects of delayed graft function and cold ischaemia time on renal allograft survival. Nephrol Dial Transplant. 2006; 21:1689-96.

54. Elia N, Tramer MR. Ketamine and postoperative pain-a quantitative systematic review of randomized trials. Pain. 2005; 113:61-70.

55. Jadad AR, Moore RA, Carroll D, Jenkinson C, Reynolds DJ, Gavaghan DJ, McQuay HJ. Assessing the quality of reports of randomized clinical trials: is blinding necessary? Control Clin Trials. 1996; 17:1-12.
56. Veroniki A, Salanti G. Graphical exploration of inconsistency in a network of randomized trials in R., http:// www.mtm.uoi.gr/index.php/how-to-do-an-mtm/10-how-todo-an-mtm/18-inconsistency; 2012.

57. Spiegelhalter DJ, Best NG. Bayesian approaches to multiple sources of evidence and uncertainty in complex costeffectiveness modelling. Stat Med. 2003; 22:3687-709.

58. Salanti G, Ades AE, Ioannidis JP. Graphical methods and numerical summaries for presenting results from multipletreatment meta-analysis: an overview and tutorial. J Clin Epidemiol. 2011; 64:163-71.

59. Begg CB, Mazumdar M. Operating characteristics of a rank correlation test for publication bias. Biometrics. 1994; 50:1088-101. 